FORMATION Formation emploi

Revue française de sciences sociales

116 | octobre-décembre 2011

Pêle-mêle

\title{
Les politiques d'insertion : quelle pertinence pour les « inemployables »?
}

Inclusion policies: of what relevance to the "unemployable"?

Eingliederungspolitik: wie sachdienlich ist sie für Nichtbeschäftigungsfähige?

Las políticas de inserción : ¿cuál es su pertinencia para los "inempleables"?

\section{Thérèse Levené}

\section{(2) OpenEdition}

Journals

Édition électronique

URL : http://journals.openedition.org/formationemploi/3472

DOI : 10.4000/formationemploi.3472

ISSN : 2107-0946

\section{Éditeur}

La Documentation française

Édition imprimée

Date de publication : 30 décembre 2011

Pagination : $51-67$

ISSN : 0759-6340

\section{Référence électronique}

Thérèse Levené, «Les politiques d'insertion : quelle pertinence pour les « inemployables » ? »,

Formation emploi [En ligne], 116 | octobre-décembre 2011, mis en ligne le 11 janvier 2014, consulté le 30 octobre 2020. URL : http://journals.openedition.org/formationemploi/3472 ; DOI : https://doi.org/ 10.4000/formationemploi.3472 


\title{
Les politiques d'insertion : quelle pertinence pour les « inemployables »?
}

Thérèse Levené

\begin{abstract}
On peut s'interroger sur la pertinence des programmes proposés aux adultes en parcours d'insertion; d'autant plus que la circulation de l'un à l'autre des dispositifs, les attentes et les espoirs révèlent un décalage entre les intentions politiques et les moyens mis en œuvre.
\end{abstract}

La notion d'insertion apparaît, au début des années 70, dans les rapports des commissions des $\mathrm{V}$ et $\mathrm{VI}^{\mathrm{ème}}$ plans. Elle désigne alors les réponses étatiques au chômage des jeunes et à "la nouvelle pauvreté ${ }^{2}$. Vingt ans plus tard, elle sera promue au rang d'impératif national visant alors «les exclus $»^{3}$ (Mauger,

\footnotetext{
${ }^{1}$ L'article proposé rend compte d'une partie des résultats issus d'une recherche conduite par l'auteure avec le soutien de la Fondation Orange dans le cadre d'un mécénat soutenant la lutte contre l'illettrisme. Cette recherche a donné lieu à un rapport : Levené T., Dubus A. (2010). Expériences, parcours, contextes résidentiels et rapports à la formation des adultes peu qualifiés. http://halshs.archives-ouvertes.fr/halshs-00529887/fr/

${ }^{2}$ Le terme " nouvelle pauvreté » est apparu dans les années 80 . Les « nouveaux pauvres » désignent les personnes fragilisées par le chômage de longue durée ou la précarité des emplois dans les sociétés industrialisées. La pauvreté est définie par rapport aux revenus, on parle de « seuil de pauvreté »; il est question de « travailleurs pauvres » ou « d'étudiants pauvres ».

${ }^{3}$ Dans le langage courant, le terme d'exclus renvoie à ceux qui ne participent plus à la production des richesses et qui n'en reçoivent plus les bénéfices. On doit notamment à Paugam (2000) une approche sociologique du "processus d'exclusion » qui consiste en une marginalisation progressive : la privation prolongée d'emploi ou une situation d'emploi précaire rend vulnérable et met peu à peu à l'écart ; l'absence de reconnaissance sociale peut s'accompagner
}

Thérèse Levené est maître de conférences en sciences de l'éducation au Centre inter-universitaire de recherches en éducation de Lille (CIREL), équipe Trigone. Ses recherches portent sur la formation des adultes dans une approche sociologique des acteurs. L'auteure a publié récemment: Levené Th., Vernies Ch. (2008), "L'évolution des motivations de stagiaires engagés dans une démarche d'insertion socioprofessionnelle », Transformations - recherches en éducation des adultes, $n^{\circ} 1$, pp. 49-67; Levené Th. (2009), "Insertion et rapports au temps ». VEl-Diversité, $n^{\circ} 158$, pp. 73-79. Levené Th., Bros F. (2009). Parcours d'insertion et engagement en formation des adultes peu qualifiés et peu scolarisés. Actes du colloque international francophone de l'association Recherches et pratiques en didactique professionnelle, 2-3-4 décembre 2009, AGROSUP, Dijon. www. didactiqueprofessionnelle.org Levené Th., Bros F. (2010), Contextes résidentiels et rapports à la formation d'adultes faiblement scolarisés/qualifiés. Congrès international Actualité de la Recherche en Education et Formation (AREF), AECSE, SSRE, SGBF, ABC Educ, Genève (Suisse). 
2001), considérés pour partie "inemployables 》 (Ebersold, 2001).

L'histoire de l'insertion se caractérise par deux âges marqués par deux perspectives et deux logiques d'intervention sur le non-emploi et sur ses conséquences (Mauger, 2001). D'une part, les politiques publiques des années 70 aux années 90 sont centrées sur l'accès à l'emploi salarié et le développement de la formation professionnelle. Cette perspective éducative place l'alternance au cœur de la démarche d'insertion professionnelle. D'autre part, le début des années 90 se focalise sur les conséquences sociales du chômage et privilégie le développement de formes économiques alternatives s'appuyant notamment sur l'économie dite solidaire. Cette perspective économiste s'inscrit dans une dérégulation programmée du marché de l'emploi. Elle place le sans-emploi mais aussi le salarié dans un processus de construction et d'entretien permanent de leur légitimité professionnelle. Ils doivent entretenir ou développer leur « employabilité ».

Ce néologisme, qu'est l'employabilité, est le maîtremot du deuxième âge de l'insertion mais aussi de l'ère du management ou des nouveaux rapports de travail, caractérisés par la flexibilité du marché de l'emploi. L'employabilité doit être comprise comme la capacité à trouver un employeur à qui le travailleur apporte pour un temps son «capital humain», de façon à réaliser "une combinaison productive » rentable (Dubar, 2004). Le terme, d'abord relevé en 1978 dans un document de l'OCDE puis dans le Livre blanc de la Commission européenne Vers la société cognitive $^{4}$, a connu sa consécration juridique avec son apparition dans la loi du 13 février 2008 relative au service public de l'emploi'.

Le recours à ce terme suggère que certains seraient employables quand d'autres ne le seraient pas ou plus. Le chômage serait dorénavant imputé à « l'inemployabilité » d'une partie de la population active. L'inemployabilité est une notion très peu usitée. On ne parle pas d'inemployables mais de «personnes

du développement d'un sentiment d'inutilité, voire d'incompétence, qui conduit à la perte d'identité, sans plus aucune place assignée dans la société.

${ }^{4}$ http://europa.eu/documents/comm/white_papers/pdf/ com95_590_fr.pdf

${ }^{5}$ Loi n ${ }^{\circ} 008-126$ du 13.02.2008, article 16. Code du travail, article L 5312-1. les plus éloignées de l'emploi », et parmi elles la distinction est faite entre les "très éloignés » et ceux qui le sont moins, sur une base non clairement définie (Hagneré, 2004). Les mesures d'insertion professionnelle s'inscriraient dans la restauration ou le développement de l'employabilité pour les moins éloignés des éloignés de l'emploi, tandis que l'insertion sociale contribuerait à "l'activation des potentialités et des ressources ${ }^{6}$ des handicapés sociaux.

Les mesures destinées à cette seconde catégorie et leur opérationnalisation sur le terrain font l'objet de cet article : quels traitements sont réservés aux « inemployables »?

Après une rapide présentation des dispositifs mis en place à l'attention des publics éloignés de l'emploi, nous découvrirons l'offre d'insertion à destination des plus éloignés de l'emploi d'entre eux en régions Nord Pas de Calais et Champagne Ardenne. Précisément, nous interrogerons la pertinence de cette offre en termes de développement ou de recouvrement d'une employabilité au regard des caractéristiques des bénéficiaires, de leurs expériences scolaires et de formation, de leurs attentes et du contexte d'emploi.

\section{LES MESURES D'AIDE À L'EMPLOI ET LEURS BÉNÉFICIAIRES}

Les mesures d'aide à l'emploi prennent plusieurs formes ; parmi elles, nous nous intéresserons aux stages de réadaptation socioprofessionnelle. Quatre programmes ou dispositifs ont été répertoriés. Ils ont des vocations différentes et ne répondent pas aux mêmes besoins ; ils sont cependant mis en place, la plupart du temps, au sein des mêmes organismes de formation dont le projet est l'aide à l'insertion socioprofessionnelle des adultes peu qualifiés. Lors de nos prises de contacts avec les organismes de forma-

\footnotetext{
${ }^{6}$ "L'activation des potentialités et des ressources » : nouveau référentiel en travail social ; il s'agit désormais de substituer aux mécanismes assistantiels de l'État providence dits passifs, des mécanismes plus incitatifs qui inviteraient l'individu accompagné à se prendre en main, à définir son projet, à construire son autonomie, mesurée in fine à l'aune de son insertion sur le marché de l'emploi. Voir notamment à ce sujet les travaux de M. Alaluf (2000), d'A. Franssen (2008) ou encore de R. Castel et l'article de Bartholémé et Vranken (2005).
} 
tion, nous avons observé que d'anciens stagiaires de programme de mobilisation ou d'orientation suivaient une formation de remise à niveau en maîtrise des savoirs de base (MSB) ou, qu'à l'inverse, des stagiaires en définition de projet professionnel provenaient de MSB ; nous avons donc avancé l'hypothèse d'une circulation entre ces dispositifs. Les données recueillies ont permis de valider cette hypothèse.

\section{Contrats aidés et stages}

Le recours aux stages et contrats aidés pour l'insertion des publics en difficulté sur le marché du travail constitue un élément très important de la politique de l'emploi, en France, depuis le début des années 90. En 2008, 29,8 milliards ont été dépensés au titre de la formation professionnelle et de l'apprentissage en direction des demandeurs d'emploi et des actifs occupés. La même année, l'État et les régions ont investi 2,25 milliards pour la formation des demandeurs d'emplois (respectivement 911 millions et 1,332 milliard), rémunérations comprises (Delort, 2010).

Les mesures d'aide à l'emploi prennent quatre formes : des aides à l'embauche en entreprise sous forme de contrats aidés marchands ; des contrats aidés non marchands incitant les associations ou collectivités locales à répondre à des besoins collectifs en recrutant les personnes les plus exposées au chômage ; des contrats de formation en alternance ou contrats de professionnalisation; enfin, des stages de formation pour demandeurs d'emploi. Les contrats aidés marchands ou non marchands, les contrats de formation en alternance ou les contrats de professionnalisation prévoient des temps de formation; ils relèvent du code du travail.

Les stages de formation, quant à eux, sont des stages de réadaptation sociale et professionnelle organisés par les conseils régionaux ${ }^{7}$. La loi de programmation pour la cohésion sociale, du 18 janvier 2005, laisse à ces derniers une certaine initiative quant au mode de mobilisation des publics prioritaires. Ces stages offrent des activités de socialisation ou de remise à

\footnotetext{
${ }^{7}$ En 2008, les dépenses des régions liées aux stages de formation des demandeurs d'emplois s'élevaient à 504 millions d'euros, les dépenses liées aux stages de formation des jeunes représentaient 435 millions d'euros (rémunérations non comprises dans les deux cas), soit $21 \%$ des dépenses des conseils régionaux en matière de formation professionnelle (Delort, 2010).
}

niveau en alternance avec des périodes en entreprise. Le stage en entreprise demeure le pivot de la formation, comme au cours de la première ère de l'insertion, mais la formation n'est plus qualifiante.

La population qui nous intéresse est inscrite dans ces stages. Elle est peu qualifiée ${ }^{8}$ au sens où la majeure partie de ses membres a quitté le système éducatif à l'issue de la scolarité obligatoire sans diplôme et/ou n'a occupé que des emplois au bas de la hiérarchie des qualifications. Elle est composée de jeunes, de mères de famille, de bénéficiaires de minima sociaux, de chômeurs indemnisés, de personnes handicapées ou encore de salariés dont la carrière est menacée en raison de faibles acquisitions scolaires. Ces personnes ont en commun une grande difficulté à trouver (ou conserver) un emploi durable.

Une recherche menée en 2009 (Levené \& Dubus, 2010) a mis à jour les caractéristiques et la diversité de ce groupe social très peu médiatisé qui participe, volontaire ou contraint, à des programmes de réadaptation socioprofessionnelle.

\section{Les stages de réadaptation sociale et professionnelle}

\section{Une organisation autour de quatre programmes}

L'offre de formation à destination des adultes sans qualification s'articule autour de quatre programmes : les dispositifs dits de maîtrise des savoirs de base (MSB), destinés aux personnes en situation d'illettrisme ; les actions de socialisation ou mobilisation à destination des jeunes de moins de 25 ans ; les formations à visée d'orientation accompagnant à la définition d'un projet professionnel et enfin les formations pré-qualifiantes. Ces formations, d'une durée de 3 à 6 mois, ne délivrent pas de diplôme.

Au moment de l'enquête, parmi les 1076 répondants, près de $66 \%$ sont inscrits dans des actions visant le (ré)apprentissage des savoirs de base ; $19 \%$ fréquentent des actions dites de « mobilisation » à vocation de dynamisation et de socialisation (dispositif Horizons en Nord Pas de Calais ou Ecole de la deuxième

\footnotetext{
${ }^{8}$ On distinguera, avec Rose (2009), la personne de l'emploi et même du travail dits non qualifiés.
} 


\section{Encadré 1}

\section{Méthodologie}

Une enquête sous forme de questionnaire a été adressée à des adultes peu ou pas qualifiés et inscrits en centres de formation en Nord Pas de Calais et Champagne Ardenne. Le choix de ces deux régions repose d'abord sur la bonne connaissance du terrain des chercheurs de l'équipe Trigone du Centre inter-universitaire de recherches en éducation de Lille (CIREL), qui mènent depuis de nombreuses années des recherches sur l'illettrisme et les problématiques d'insertion des adultes peu ou pas qualifiés. De plus, ces deux régions présentent des similitudes sur les plans économique et social : les nombreuses tentatives de reconversion économique, notamment par le tourisme et le développement des services, ne parviennent pas à enrayer les effets néfastes de la crise : le taux de pauvreté atteint 15,8 \% dans le département des Ardennes, juste après le Nord (16,6\%) et le Pas-deCalais (16,7\%). Le niveau de vie médian, inférieur à 15069 euros pour un ménage, demeure, dans ces trois départements, parmi les plus faibles de France avec les départements de la Corse et du Languedoc-Roussillon.

34 centres de formations ont participé à l'enquête. Les enquêteurs, chercheurs et étudiants, se sont déplacés dans 47 sites implantés dans 13 des 15 zones d'emploi du Nord Pas de Calais et 5 des 8 zones d'emploi de Champagne Ardenne. Ces sites ont été choisis en fonction de leur répartition géographique dans une tentative de représentativité de la distribution de l'offre. 1076 stagiaires ont accepté de participer à l'enquête. La passation directe des questionnaires par groupe de 10 à 30 personnes a enrichi cette enquête de données qualitatives consignées dans les journaux de bord des enquêteurs : impressions relatives à l'ambiance, au climat de la passation (niveau d'attention du groupe, adhésion ou rejet vis-à-vis de la démarche d'enquêtel ; informations relatives au cadre pédagogique de la formation lagencement spatial et logistique de la formation, affichages, supports d'apprentissage); attitudes et réactions spontanées manifestées par les enquêtés; parole des formateurs, etc.,...

L'enquête s'est déroulée en deux temps, de janvier à juillet 2009. Le questionnaire comporte trois parties. Dans la première partie, les enquêtés sont invités à se prononcer sur un certain nombre de propositions relatives à la formation, l'emploi et le chômage. Cette partie leur a été présentée à deux reprises en début et en fin de formation. II s'agissait de mettre à jour une éventuelle évolution de leurs représentations et de la nature de leur engagement en formation.

La seconde partie vise à recueillir des données relatives aux conditions de vie des enquêtés, à leurs expériences scolaires ou de formation, professionnelles, culturelles et géographiques afin de mettre à jour leurs caractéristiques spécifiques.

La troisième partie prend la forme d'une question ouverte invitant les enquêtés à faire état de leurs parcours professionnel et de formation depuis la fin de leur scolarité $\left({ }^{*}\right)$.

(*) Les enquêteurs ont lu les questions aux enquêtés en situation d'illettrisme, ces derniers cochaient eux-mêmes leur réponse sur une échelle de valeur. Les quelques réponses aux questions ouvertes ont été écrites par les enquêteurs sous la dictée des répondants. 
chance en Champagne Ardenne) ; $12 \%$ participent à des actions d'orientation; enfin, 3,5\% suivent une formation pré-qualifiante.

\section{Un parcours sans fin}

Pour plus d'un enquêté sur deux, la formation en cours n'est pas la première : $51 \%$ déclarent avoir déjà suivi au moins un des stages de formation parmi les quatre de l'offre.

Près d'un quart a déjà suivi au moins une formation dans le même centre de formation et $38 \%$ dans un autre centre de formation.

Près de la moitié des stagiaires de MSB a déjà entrepris une remise à niveau. On peut considérer qu'ils poursuivent un travail engagé : le dispositif prévoit une formation en trois étapes correspondant chacune à un niveau de maîtrise des compétences en lecture ou écriture ${ }^{9}$. La réinscription fait donc partie du processus de formation.

On relève qu'un quart des inscrits en pré-qualification « repique » et que $5 \%$ des stagiaires en programmes de mobilisation ou d'orientation refont le même stage. Ces « redoublements » peuvent s'expliquer par le besoin d'un temps d'accompagnement plus long pour certains. La circulation d'un dispositif à l'autre, dont il est peu question sur le terrain, a retenu notre attention.

Le passage d'un dispositif à l'autre pourrait, dans certains cas, avoir du sens ; cependant, si l'on considère la dernière formation déclarée ${ }^{10}$, la configuration de cette circulation apparaît quelque peu inattendue.

Le dispositif de maîtrise des savoirs de base s'adresse aux personnes en situation d'illettrisme ou en déficit d'apprentissages scolaires de base ; la formation dite de pré-qualification - par définition préparatoire à une formation qualifiante - se situe en principe à

\footnotetext{
${ }^{9}$ Le niveau $1 \mathrm{~s}$ 'adresse aux non-lecteurs ou aux faibles lecteurs ; le niveau 2 à des personnes sachant lire mais éprouvant d'énormes difficultés à produire un texte compréhensible; le niveau 3 concerne des lecteurs qui produisent un écrit comportant beaucoup d'erreurs : http://cueep.univ-lille1.fr/fichecueep/GroDoc/CataDispoSignet. pdf

${ }^{10}$ Les réponses relatives aux formations antérieures n'ont pas été fournies avec précision, contrairement aux parcours biographiques auxquels les enquêteurs ont été particulièrement attentifs. Les enquêtés ont généralement cité une seule formation. L'analyse des parcours a montré qu'il pouvait y en avoir eu à plusieurs reprises dans une même trajectoire...
}

un niveau de qualification supérieur à celui requis pour entrer en MSB ; les actions de mobilisation s'adressent aux jeunes « démobilisés » réunis autour d'un projet collectif, culturel, sportif, ou humanitaire censé les rendre acteurs de leurs parcours d'insertion professionnelle ; enfin, les programmes orientation concernent les plus qualifiés, déjà expérimentés et ayant besoin de soutien pour clarifier leurs aspirations et compétences.

Ceci suggérerait que «mobilisation » pourrait précéder n'importe quel autre dispositif et que logiquement «MSB » devrait précéder plutôt que suivre " pré-qualification » et « orientation ». Or, pratiquement, l'ordre inverse est observé.

De 10 à $12 \%$ des stagiaires inscrits en MSB, en programme orientation ou en mobilisation sont issus d'une formation pré-qualifiante. La pré-qualification ne parvient pas, pour ces derniers, à amorcer le processus de qualification : niveau trop peu élevé des bénéficiaires, pédagogie mal adaptée à une population adulte ? La pré-qualification viendrait constater le besoin de réapprentissage de certains, le défaut de mobilisation des plus jeunes ou l'absence de projet des plus âgés, ceux qui rejoignent le dispositif d'orientation. La présence en MSB d'anciens inscrits en mobilisation ou en programme d'orientation (respectivement 5 et $4 \%$ ) interroge également la cohérence de la circulation et la fonction des dispositifs :

- le passage en mobilisation a pu permettre le repérage d'une situation d'illettrisme; dans ce cas, l'accueil en MSB est pertinent ;

- le temps de la mobilisation n'a pu déboucher sur une insertion professionnelle ou offrir l'accès à une formation qualifiante ; dans ce cas, la présence en maîtrise des savoirs de base est une solution d'attente "tolérée par la Région" ", confie une formatrice qui ajoute : «on peut pas les lâcher sans rien!».

- la participation à une formation en MSB ou à une action de mobilisation après un programme de définition de projet professionnel ( $6 \%$ de ces derniers) remet non seulement en cause la validité de l'orientation initiale mais soulève clairement la question de l'accueil du marché de l'emploi...

${ }^{11}$ ce qui signifie que la région accepte de financer la formation. 
Ce premier état des lieux révèle un dispositif central « très accueillant », ouvert aussi bien à ceux à qui les programmes de pré-qualification, de mobilisation ou d'orientation n'ont pas offert de débouchés qu'à des adultes immigrés analphabètes ou diplômés. La présence de ces derniers en MSB sera expliquée plus loin.

Les stages de formation à destination des adultes peu ou pas qualifiés poursuivent en principe des objectifs distincts ; cependant, ils constituent de fait, pour une part de la population, des étapes successives et non ordonnées d'un parcours d'insertion pas vraiment balisé et dont les perspectives demeurent incertaines. Certains s'y installent durablement faute d'insertion professionnelle ou alternent contrats aidés, petits boulots et stages de formation, comme on le verra plus loin dans l'analyse des trajectoires.

Ces programmes peuvent-ils répondre aux besoins de formation et d'insertion que révèlent les expériences professionnelles et scolaires et les attentes de leurs bénéficiaires?

\section{LES BÉNÉFICIAIRES DES STAGES DE RÉADAPTATION}

Nous allons à présent nous intéresser aux caractéristiques des stagiaires, précisément à leurs statuts, leurs trajectoires professionnelles et leurs parcours scolaires. Il s'agit de mieux connaître leurs expériences et leurs besoins.

\section{Jeunes, chômeurs indemnisés, bénéficiaires du RMl et des salariés}

Les stagiaires des dispositifs d'insertion sont jeunes, avec un âge médian de 23,5 ans. Un quart de la population ne dépasse pas l'âge de 19 ans. La présence exclusive de « jeunes » en programmes de mobilisation est une explication, leur nombre en MSB n'est pas négligeable : un quart d'entre eux ne dépasse pas 21 ans, près de $2 \%$ sont âgés de 16 ans et près de $3 \%$ de 17 ans. Des jeunes en situation d'abandon scolaire sont envoyés par les missions locales en stage de remise à niveau dès leur sortie d'école... Leur présence en formation leur vaut une indemnité versée par le CNASEA ${ }^{12}$.

La formation constitue ainsi, dans certains cas, la garantie d'une forme d'assistance qui se substitue au salaire (Bec, 1999) : elle peut faire partie des clauses du contrat signé par les bénéficiaires du revenu minimum d'insertion ( $14 \%$ de la population) et être même une condition du maintien de l'allocation. Elle consiste parfois en une obligation pour certains chômeurs indemnisés (14\%). 4,4 \% de la population perçoit l'allocation pour adulte handicapé, $9 \%$ de la population ne déclare aucun revenu.

Ils sont peu nombreux, cependant, à considérer leur inscription en formation comme une obligation.

On compte 155 personnes salariées, toutes inscrites en MSB ; elles représentent $14,5 \%$ de la population et près de la moitié d'entre elles relève du programme " clés de la formation », destiné aux bénéficiaires de contrats aidés.

\section{Une expérience professionnelle non valorisée}

\section{Des emplois non qualifiés}

Bien que près de $86 \%$ des répondants déclarent être sans emploi au moment de l'enquête, $58 \%$ ont exercé au moins un métier et $67 \%$ de ceux qui ont déjà travaillé ont exercé plusieurs métiers. Ces métiers appartiennent, dans l'ordre, aux services aux entreprises et collectivités (entretien des locaux, entretien des espaces verts et sécurité) avec $29 \%$ des derniers emplois déclarés, à l'industrie de fabrication $(24 \%$ des emplois), au secteur des services aux particuliers (restauration, coiffure) avec $14 \%$ des derniers emplois déclarés. Viennent ensuite le secteur du Bâtiment et Travaux Publics avec $13 \%$ des emplois, les services à la personne $(11 \%)$ et le commerce $(11 \%)$. Les

${ }^{12}$ Le Centre national pour l'aménagement des structures des exploitations agricoles est un établissement public placé sous la tutelle des ministères de l'Agriculture et de la Pêche, de l'Emploi et des Affaires sociales. Il a fusionné avec l'Agence unique de paiement le $1^{\text {er }}$ avril 2009 pour former l'Agence de services et de paiement. Le CNASEA intervient dans deux domaines principaux : l'agriculture (les programmes de développement et de mise en valeur du monde rural) et la formation professionnelle et l'emploi, notamment par le paiement des mesures en faveur de l'emploi (Contrats aidés, stages de formation). 
Tableau 1

Répartition de la population selon les trajectoires professionnelles

\begin{tabular}{|l|c|c|}
\cline { 2 - 3 } \multicolumn{1}{l|}{} & Effectifs & $\% /$ Total \\
\hline " Anciens » expérimentés & 93 & $9,0 \%$ \\
\hline Jeunes diplômés en « galère » & 140 & $13,6 \%$ \\
\hline Chômeurs longue durée & 153 & $14,8 \%$ \\
\hline Mères de famille & 101 & $9,8 \%$ \\
\hline Jeunes en Formation & 105 & $10,2 \%$ \\
\hline Jeunes en recherche d'un premier emploi & 145 & $14,00 \%$ \\
\hline Jeunes sans activité particulière & 250 & $24,3 \%$ \\
\hline En graves difficultés & 44 & $4,3 \%$ \\
\hline Total & 1031 & $100.00 \%$ \\
\hline
\end{tabular}

$\mathrm{n}=1031$, soit 45 non réponses.

emplois occupés sont des emplois non qualifiés dans des secteurs connus pour leur recrutement de travailleurs non qualifiés (Rose 2009, p. 35). Il s'agit d'emplois faiblement rémunérés, non valorisés socialement (Rose, p. 12), souvent à temps partiel contraint, qui maintiennent les salariés dans un état de grande précarité.

Des trajectoires professionnelles liées à la conjoncture économique

Nous distinguerons plusieurs types de trajectoires professionnelles : les « anciens » expérimentés, les jeunes diplômés en "galère », les chômeurs longue durée, les mères de famille, les jeunes en formation, les jeunes en recherche d'un premier emploi, les jeunes sans activité particulière, les personnes «en graves difficultés $»$.

Les anciens non qualifiés et les jeunes « diplômés » entretiennent des liens avec le monde du travail

Ceux qui ont connu (ou connaissent encore) une situation professionnelle relativement stable sont parmi les plus âgés et les moins qualifiés : ils représentent $9 \%$ de la population. Ils n'ont pas toujours fréquenté le collège et quand ils l'ont fréquenté, ils n'ont pas obtenu le brevet des collèges. C'est en MSB qu'on les rencontre.

À l'opposé, par l'âge et les expériences cumulées, des jeunes souvent titulaires de CAP et surreprésentés en programme Orientation cumulent petits boulots ou emplois à durée déterminée et temps partiel, qualifiés pour certains, qui n'offrent pas une insertion professionnelle durable (14\%).

Ces deux groupes sont victimes du contexte économique général défavorable; les premiers peinent à se réinsérer en raison notamment de leur faible niveau de qualification initiale et leur absence de formation professionnelle ; les seconds appartiennent à la génération "galère » (Alternatives économiques, mars 2011).

Les plus éloignés de l'emploi : des hommes entre $\mathbf{2 0}$ et $\mathbf{4 0}$ ans et des mères de famille

Les chômeurs de longue durée (15\%) sont plutôt des hommes ; ils ne présentent pas de signes particuliers 
en termes de qualification au regard de la population enquêtée ; ils se distinguent toutefois par des expériences de travail de courte durée, entrecoupées de stages de formation et particulièrement de stage de pré-qualification. Ils ont même épuisé leurs droits aux indemnités du service public de l'emploi ; ils sont en majorité prestataires du RMI.

Des mères de famille (10\%) souhaitent acquérir ou développer des savoirs de base en vue d'une insertion dans le monde du travail. Certaines ont travaillé avant de se consacrer à leurs enfants, malgré une absence de diplôme, ou ont effectué des tentatives infructueuses de remise au travail.

\section{— Les jeunes non qualifiés}

Un groupe passe son temps en formation depuis sa sortie de l'école, que ce soit en MSB, mobilisation ou orientation (10\%). Il rassemble les plus jeunes (moins de 21 ans en moyenne) ; beaucoup ont suivi un enseignement technique sans obtenir de diplôme supérieur au brevet des collèges.

Un autre groupe (14\%) tente une mise au travail sans succès depuis sa sortie assez récente du système scolaire. Il est composé de jeunes de 22 ans en moyenne, inscrits pour la première fois en programme de mobilisation. Comme les précédents, ils ont pour la plupart suivi des études techniques sans être plus diplômés que le reste de la population.

Les jeunes de ces deux premiers groupes vivent chez leurs parents.

Enfin, un troisième groupe (24 ans d'âge moyen), comptant un peu plus de femmes que les deux premiers, ne déclare pas d'activités particulières. Ils n'ont rien livré qui relèverait d'activités professionnelles reconnues ou de prise en charge particulière comme un stage de formation (24\%). Ils ont rejoint les précédents en «mobilisation ». Près de $60 \%$ d'entre eux vivent encore chez leurs parents.

\section{- Le cumul de graves difficultés pour une minorité}

Ces enquêtés constituent une classe peu nombreuse $(4,5 \%)$ qui se distingue par des situations sociales complexes en rupture avec l'école, l'emploi ou la formation, faites d'errance, d'abandon du domicile familial, de « dépression »... Encore jeunes, pour certains, avec un âge moyen de 26 ans et un écart type de 9,5, indifféremment hommes ou femmes, ils n'ont généralement pas atteint le collège ; quelquesuns parmi eux ont suivi un enseignement spécialisé. On les rencontre dans tous les dispositifs, sauf en préqualification et ils comptent parmi les plus anciens en formation (cinq trimestres en moyenne).

Les trajectoires professionnelles révèlent le poids du contexte socio-économique. Elles rappellent, si nécessaire, que l'insertion professionnelle dépend étroitement des contextes d'emploi et de leurs modes de gestion (Dietrich, Jouvenot, Lenain, 2007). Ces trajectoires font suite à un cursus scolaire rarement qualifiant qui a laissé peu de bons souvenirs.

\section{Des parcours scolaires rarement qualifiants}

Le temps passé à l'école est un temps riche d'expériences, non seulement en termes d'apprentissage mais aussi en termes de socialisation. Ces expériences seront autant de ressources mobilisables dans une démarche de formation d'adultes. Elles peuvent constituer des leviers ou des freins dans l'engagement en formation.

Parmi les répondants, $7 \%$ n'ont pas dépassé le primaire, $27 \%$ n'ont pas été au-delà de la troisième, $40 \%$ ont suivi tout ou partie d'une formation préparatoire à un CAP ou un BEP (certificat d'aptitude professionnelle et brevet d'études professionnelles), $16 \%$ ont fréquenté le lycée, $1 \%$ ont entamé un premier cycle universitaire ; enfin, $17 \%$ ont bénéficié d'un enseignement spécial dont plus de la moitié en institut médico-éducatif.

$89 \%$ des enquêtés ont effectué leur scolarité en France (en Belgique ou dans les DOMTOM), $11 \%$ ont effectué leur scolarité à l'étranger, dont une grande majorité hors Union européenne, la quasi-totalité de ces derniers est inscrite en maîtrise des savoirs de base.

Les répondants qui déclarent une scolarité hors de France constituent deux sous-populations aux caractéristiques antagonistes : l'une non diplômée voire non scolarisée ; l'autre titulaire du Bac ou de diplôme de l'enseignement supérieur. La première population relève de dispositifs d'alphabétisation, la seconde de cours de français langue étrangère (FLE); à défaut de 
l'existence de ces dispositifs, sur certains territoires, ces sous-groupes se retrouvent en MSB.

L'âge moyen de fin de scolarité est de 16,7, avec un écart type de 2,3 . Une partie de la population a quitté l'école avant l'âge légal. Certes, ce sont les étrangers qui affichent l'âge moyen de scolarité le plus bas mais certains français (ou européens) ont quitté l'école avant l'âge de 16 ans, comme l'indique l'écart type de cette sous-population, soit 1,95, et la valeur du premier quartile : 15 ans et demi.

Les plus âgés ont quitté l'école le plus tôt, mais l'âge moyen de fin de scolarité tend actuellement à baisser chez les plus jeunes de la population.

C'est sans doute en termes de décrochage scolaire que la problématique des non-qualifiés pourrait être posée si l'on retient la définition proposée par Janosz, Leblanc et Boulerice (1998) : "Un décrocheur est défini comme tel si il a quitté l'école sans avoir obtenu de diplôme de niveau secondaire [...] et sans non plus y être retourné pour en compléter les exigences avant l'âge de 22 ans ». Phénomène ancien, le décrochage expose, dans un contexte difficile d'accès à l'emploi, aux risques de troubles personnels ou d'entrée dans la délinquance (Janosz, 2000, Roché, 2001). Autant de difficultés à prendre en compte aussi bien dans les programmes de mobilisation qu'en maîtrise des savoirs de base. Les formateurs ont fait état, au cours de l'enquête, de la dimension avant tout éducative de leur mission.

Le parcours scolaire a pu être clôt par un diplôme. $20 \%$ sont titulaires d'un CAP ou d'un BEP relevant de spécialités offrant peu de débouchés (voir infra). Près de $3 \%$ déclarent un Baccalauréat. Ces diplômés sont surreprésentés en programme « Orientation ». 8, 5\% déclarent un brevet des collèges. $10 \%$ des répondants sont titulaires du Certificat de formation générale qui a été obtenu, pour certains, à la fin du niveau $3 \mathrm{du}$ parcours MSB.

Près de $80 \%$ des bénéficiaires des stages de réadaptation sociale et professionnelle affichent donc un niveau inférieur ou égal au niveau $\mathrm{Vbis}^{13}$.

${ }^{13}$ Niveau VI : sortie du premier cycle du second degré ( $6^{\text {ème }}, 5^{\text {ème, }}$ $\left.4^{\text {ème }}\right)$ et des formations préprofessionnelles en un an. Niveau $\mathrm{V}$ bis : sortie de $3^{\text {ème }}$ générale, $4^{\text {ème }}$ et $3^{\text {ème }}$ technologique et des classes des seconds cycles courts professionnels avant l'année terminale.
Les jeunes titulaires du brevet des collèges sont surreprésentés dans les dispositifs de mobilisation. Le brevet clôt pour eux la scolarité ; il n'offre cependant pas une qualification reconnue sur le marché du travail : «A peine plus de la moitié d'entre eux ont un emploi au bout de trois années passées sur le marché du travail, contre plus des deux tiers des jeunes sans qualification venant de l'enseignement professionnel », révélait l'enquête Génération 98 du Céreq, et de manière générale, «les jeunes ayant arrêté leurs études au niveau du collège connaissent des parcours d'insertion plus difficiles que ceux ayant débuté un CAP ou un BEP» (Gasquet, 2003).

Si les non-diplômés sont surreprésentés parmi les stagiaires inscrits en MSB, notons que $39 \%$ des bacheliers fréquentent ce dispositif et $55 \%$ des titulaires de CAP. La présence de ces derniers en MSB soulève de nombreuses questions relatives à l'obsolescence de certains diplômes ou à leur valeur insuffisante sur le marché du travail ; elle interroge de nouveau le rôle dévolu à ces dispositifs. Comment une offre de formation comme celle visant la maîtrise des savoirs de base peut-elle prendre en compte les besoins de " diplômés » qui ne parviennent pas à valoriser leur certification auprès d'employeurs, les besoins de jeunes « décrocheurs scolaires » et ceux d'adultes en situation d'illettrisme?

Les formations initiales professionnelles relèvent des spécialités préparant aux métiers des services aux entreprises et aux collectivités (25\%), du secteur industriel (travail sur chaînes automatisées) pour $22 \%$, du bâtiment avec $15 \%$ des effectifs, du secteur tertiaire marchand (commerce essentiellement) avec $11 \%$ des formations et enfin des services non marchands (petite enfance ou services à la personne) pour $10 \%$.

Les deux premières spécialités appartiennent au « trou noir » de l'insertion : les jeunes qui ont suivi ces cursus « ont sédimenté les handicaps tout au long de leurs parcours scolaires, ils éprouvent de façon homogène les plus grandes difficultés d'accès au marché du travail » et connaissent les plus forts taux de chômage (Arrighi \& Gasquet, 2010).

L'analyse des résultats de l'enquête Génération 2004 du Céreq (Arrighi, Gasquet \& Joseph 2009) a montré que les formations aux métiers du bâtiment font partie 
des formations qui accueillent une forte proportion de jeunes ayant des difficultés scolaires au collège ; en outre, ceux-ci ont tendance à abandonner la formation, sans aller jusqu'à la certification parce qu'ils trouvent un emploi... non durable. L'enquête le confirme : les enquêtés qui déclarent une formation en bâtiment ont, à l'image des jeunes de la génération 2004, entamé une formation technique sans obtenir de diplôme. Si l'insertion professionnelle des jeunes issus de l'enseignement technique est potentiellement meilleure que celle des seuls détenteurs du brevet des collèges, c'est un emploi non qualifié qu'ils risquent d'occuper: " près de la moitié des jeunes qui sortent sans diplôme de CAP ou BEP (niveau V bis) ont, lorsqu 'ils travaillent au bout de trois ans de vie active, un emploi temporaire ou non qualifié » (Gasquet, 2003). Les jeunes rencontrés en stage n'ont cependant, pour beaucoup d'entre eux, pas réellement entamé une vie active... Le stage les y conduira-t-il ?

\section{Une expérience scolaire souvent mal vécue}

Que conservent ces adultes jeunes ou moins jeunes comme souvenir ou comme image de l'école $?^{14}$

Un tiers des répondants affiche une certaine indifférence vis-à-vis de leur expérience scolaire : ils n'ont « rien à en dire », «c'est trop loin », comme si l'école ne les avait pas concernés. Cette attitude prévaut parmi les stagiaires de MSB. Seraient-ils restés «étrangers à l'enseignement dispensé » comme Sylvain, l'un des personnages centraux de l'enquête ethnographique de Renahy (2006) sur les jeunes ouvriers ruraux, déjà « déscolarisé dans l'école » (Bautier, Terrail, BrancaRosoff et al., 2002) ?

$28 \%$ entretiennent au contraire un rapport amer à leur expérience scolaire ; ils ont le sentiment de ne pas avoir été aidés ou compris, font parfois état de moments de souffrance. On peut dire qu'ils éprouvent une certaine rancune vis-à-vis de l'école. Ce sentiment est plutôt masculin.

$18 \%$ invoquent une interruption de leur scolarité pour raison de « force majeure », beaucoup sont des femmes. Des événements comme la grossesse ou

${ }^{14}$ «Si on me demande de parler de ma scolarité, que dirais-je à ce sujet? » est-il demandé aux enquêtés. le mariage ont été relevés comme cause d'abandon scolaire. Les femmes ont tendance à exprimer des regrets d'avoir été rappelées à leurs conditions d'épouse, de mère ou de soutien de famille... Des jeunes déclarent : «y'avait trop de problèmes dans ma famille », «mon père voulait pas que je continue $»^{15}$.

$12 \%$ des répondants gardent de "bons souvenirs" de l'école ; ils sont parmi les plus qualifiés relativement ${ }^{16}$ ou inscrits en programme d'orientation : ces stagiaires encore jeunes ont le sentiment d'avoir suffisamment de ressources qu'ils attribuent à l'école. Ils se trouvent, au moment de l'enquête, dans une dynamique d'insertion active

Enfin, $9 \%$ s'estiment responsables de leur échec scolaire ; on les rencontre parmi les moins qualifiés ou les stagiaires en mobilisation; avec le recul, parfois peu important pour ces derniers, c'est à leur attitude ou à leur niveau insuffisant qu'ils attribuent leur sortie sans diplôme de l'école: "j'ai fait le con », "j'ai raté mon CAP à cause de la théorie ». S'ils ne conservent pas une bonne image de l'école car ils y ont souffert aussi, ils ne se montrent pas amers. Ils ont plutôt l'impression d'avoir loupé quelque chose : « quand on est jeune on se rend pas compte », disent les plus âgés. Discours convenu ou ébauche d'une remise en question? Une seule action de (re)mobilisation serat-elle en mesure de les aider à modifier leur rapport à l'apprentissage ou au savoir?

Réapprentissage des savoirs de base, mobilisation, orientation et pré-qualification définissent les programmes des stages de formation à destination des personnes privées d'emploi peu ou pas qualifiées. L'âge et le niveau scolaire déterminent l'accès à ces dispositifs jusqu'à un certain point ; en effet, en l'absence de débouchés professionnels, les stagiaires se réinscrivent dans la même formation ou dans une autre. Ils se trouvent ainsi pris dans un processus d' « insertion tout au long de la vie » qu'attestent certaines trajectoires faites de stages, de formations ou de contrats précaires.

En dépit ou en raison d'expériences scolaires et professionnelles difficiles, les stagiaires des disposi-

\footnotetext{
15 Propos recueillis au cours de l'enquête.

${ }^{16} \chi^{2}$ significatif au seuil $\mathrm{P}<.001$ en mobilisant la variable diplôme et significatif au seuil $\mathrm{P}<.0001$ en mobilisant la variable niveau de qualification.
} 
Tableau 2

Répartition de la population selon le rapport à l'école

\begin{tabular}{|l|c|c|}
\cline { 2 - 3 } \multicolumn{1}{c|}{} & Effectifs & $\% /$ Total \\
\hline Indifférent & 326 & $32,7 \%$ \\
\hline Rancune & 280 & $28,1 \%$ \\
\hline Force majeure & 178 & $17,9 \%$ \\
\hline Bons souvenirs & 119 & $12,0 \%$ \\
\hline "Ma faute » & 93 & $9,3 \%$ \\
\hline Total & 996 & $100.00 \%$ \\
\hline
\end{tabular}

tifs d'insertion ne vivent pas la formation sur le mode de la contrainte, même quand elle leur est imposée ; ils en attendent même beaucoup.

\section{DES ATTENTES FORTES VIS-A-VIS DE LA FORMATION}

Les sujets enquêtés ont été invités à se prononcer sur un certain nombre de propositions visant à appréhender les conditions concrètes de leur engagement en formation et le sens qu'ils donnaient à leur situation d' « apprentissage » ou d'insertion. L'analyse statistique des items a mis à jour huit dimensions ${ }^{17}$. Elles rendent compte des attentes de la population rencontrée en centres de formation.

Les uns et les autres se reconnaissent différemment dans ces rapports et avec plus ou moins d'intensité selon leurs expériences professionnelles et leur parcours scolaire.

\section{La formation : un tremplin vers l'emploi}

La formation, quelle qu'elle soit, est vécue comme un sas vers l'emploi. Ces attentes, exprimées surtout par les enquêtés les moins expérimentés, contrastent avec une réalité socio-économique qui leur offre peu de

${ }^{17}$ Ces dimensions sont construites à partir des items soumis à l'appréciation des enquêtés en $1^{\text {ere }}$ partie du questionnaire et regroupés en raison de leur corrélation statistique et de leur proximité sémantique. L'échelle des dimensions construites s'étale sur une amplitude de -1 à +1 . (Levené \& Dubus, 2010, p. 191 et suiv.). chances d'insertion. Et les espoirs sont souvent déçus, particulièrement chez les plus jeunes de la population. Les plus en difficultés, quant à eux, savent déjà, d'une certaine façon, que ce n'est pas la formation qui les « tirera d'affaire ».

\section{La formation devrait conduire à l'emploi...}

Pour certains, la formation représente déjà une forme de mise au travail, sa fonction est « occupationnelle »; pour d'autres, elle est un sas vers l'emploi, sa fonction est alors instrumentale, c'est ce que recouvre la dimension Travailler : « la formation, c'est comme un travail » ou «ça sert à trouver un travail » disent certains stagiaires. Le contenu pratique de la formation - l'expérience en entreprise - est fortement investi par les stagiaires. Ils voient, dans la situation de stage, une nouvelle (ou première) étape vers l'emploi, il s'agit ici de la dimension Espoir stage. Sur le terrain, on a pu vérifier l'importance du stage dans l'ensemble des formations : en mobilisation, le stage en entreprise est présenté comme une prise de contact avec le monde du travail et une appropriation de ses exigences et contraintes ; en orientation, le stage a valeur de test par rapport à un projet professionnel; en maîtrise des savoirs de base, le stage est proposé aux stagiaires qui «donnent des signes de volonté d'insertion professionnelle $»^{18}$.

Dans l'esprit des stagiaires rencontrés, la formation est presque l'emploi ; en tout cas, elle devrait déboucher sur un emploi. Il ne s'agit pas, en ce qui les concerne, de formation qualifiante ; pour autant, cette représentation d'un lien étroit entre formation et

${ }^{18}$ Propos recueillis auprès d'un formateur en MSB. 
emploi est bel et bien affirmée. Le danger de l'idéologie, très répandue, selon laquelle le chômage serait dû à un manque de formation, est de faire porter aux moins qualifiés la responsabilité de leur situation de chômage ; et de renforcer ainsi la dimension instrumentale de la formation, au risque de négliger les intérêts à long terme des publics peu qualifiés : acquérir non seulement une qualification professionnelle mais aussi une qualification sociale au sens de C. Demunter $(1999)^{19}$.

Des données objectives contredisent cette idéologie :

- d'une part, si le diplôme protège du chômage ${ }^{20}$, «sur 100 recrutements, seulement 10 sont le fait de primosortants de la formation initiale [et, seulement $30 \%$ des individus exercent des métiers pour lesquels ils ont suivi une formation professionnelle initiale], la position des autres dépend de leurs expériences de vie ou de leurs expériences professionnelles » (Vergne, 2006) ;

- d'autre part, l'emploi non qualifié est en progression : en 2007, on compte 3,6 millions d'employés et 1,9 million d'ouvriers non qualifiés, soit $22 \%$ de l'emploi salarié (Jauneau 2009) contre $27 \%$ en 1982 (Chardon, 2001). Ceci résulte surtout d'une forte progression des effectifs d'employés non qualifiés dans les services aux particuliers mais aussi dans les services aux entreprises en raison du développement de la logistique, de l'entretien, de la surveillance, des commerces et de la restauration. Et le secteur tertiaire a été particulièrement sensible aux mesures prises en 1993 en matière d'allègement des charges sociales sur les bas salaires.

Ces constats chiffrés révèlent les limites du raisonnement en termes d'adéquation formation-emploi. Introduisant une variable géographique, l'Observatoire des inégalités (2009) a mis en évidence qu'à niveau de diplôme égal, le taux de chômage est très supérieur dans les zones urbaines sensibles : pour les bac +2 , il atteint $13,2 \%$ contre $6 \%$ dans le reste du pays $^{21}$. Près de la moitié de la population rencontrée vit dans des communes appartenant aux proches ou

\footnotetext{
19 Prise de conscience de la place occupée dans l'organisation sociale et capacité à devenir un acteur social et politique.

$20 \mathrm{ht} \mathrm{tp://www.cereq.fr/index.php/s} \mathrm{ous} \mathrm{-} \mathrm{themes/}$ Generation-2007-Enquete-2010

21 http://www.inegalites.fr/spip.php?article314\&id_mot=87
}

grandes banlieues de villes centre au sens de Baud, Bourgeat et Bras (2008), et la majorité de celle-ci dans les bassins de Roubaix-Tourcoing et Lens-Hénin, au sud-ouest du bassin de Lille, dans le Pas-de-Calais. Le développement économique de ces deux zones a longtemps reposé sur le textile et le charbon. La reconversion économique en cours, dans ces bassins à la production diversifiée, ne se traduit pas encore par la création d'emplois accessibles aux moins qualifiés comme les enquêtés de cette recherche.

Roubaix-Tourcoing et Lens-Hénin affichent respectivement un taux de chômage de $15,4 \%$ et de $16,4 \%$ contre $11,9 \%$ pour la zone de Lille et de 13,5 \% pour la région Nord Pas de Calais. On relève une surreprésentation des « chômeurs de longue durée » et des « jeunes à la recherche d'un premier emploi » de l'enquête dans le bassin de Lens-Hénin, de même qu'une surreprésentation de jeunes avec une moyenne d'âge d'à peine 26 ans et un écart type de 10,5.

\section{... selon les moins expérimentés, à l'exception des plus vulnérables}

Les répondants sans expérience professionnelle, qui n'ont connu que le chômage, même bref eu égard à leur âge, ou qui ont passé leur temps en formation depuis la fin de leur scolarité, attendent le plus de la formation en termes d'opportunités professionnelles ${ }^{22}$. Ce n'est pas le cas de ceux qui ont connu de graves difficultés « personnelles ». Leurs très brèves expériences de travail, les longues périodes sans emploi ni indemnisation qu'ils ont connues et leur relative ancienneté dans la formation leur auraient-elles fait perdre leurs illusions ou auraient-elles développé leur lucidité ? Ils attendent peu des stages ${ }^{23}$. La formation semble être pour eux une fin en soi, un espace-temps qui les sort provisoirement de leur quotidien incertain et vide (« je ne voulais pas rester chez moi à ne rien faire »); bien sûr, ils « veulent un emploi» mais savent déjà que le marché du travail est impitoyable pour les

\footnotetext{
${ }^{22}$ Les correspondances entre les variables biographiques et les dimensions du rapport à la formation sont attestées par le test du $\chi^{2}$ ou le test F de Snedecor-Fisher.

23 Ils se situent en moyenne à 0,08 sur l'échelle de la dimension Espoir stage, dont l'amplitude va de -1 à +1 ; les jeunes peu ou pas expérimentés, un peu plus jeunes qu'eux et moins en difficultés (voir les trajectoires plus haut) se situent à 0,19 en moyenne sur cette même échelle (écart significatif, attesté par test de Fisher, au seuil $\mathrm{P}<0001)$.
} 
plus vulnérables. Leur opinion à ce sujet est d'ailleurs assez constante entre le début et la fin de leur formation, contrairement au reste de la population interrogée qui « déchante » en fin de formation. En effet, l'espoir d'une embauche s'amenuise de manière générale, et particulièrement chez les jeunes ${ }^{24}$. S'agit-il d'une désillusion? De l'intériorisation de difficultés personnelles? Ou d'une prise de conscience des effets (ou des causes) du chômage structurel ?

\section{La formation pour développer une forme d'autonomie}

La formation n'a pas pour vocation première, du point de vue des plus expérimentés sur le plan professionnel et des plus âgés, à les sensibiliser au monde du travail ; ils le connaissent déjà. Elle n'a pas pour mission de les préparer à l'emploi ; ils en ont occupé un sans qualification si longtemps... C'est souvent leur situation de chômage qui a mis à jour leur situation d'illettrisme. Ils veulent gagner en autonomie par l'apprentissage ou le réapprentissage de la lecture. Ils se montrent même avides d'apprendre et font état parfois d'une assez grande ancienneté en formation de base... Ils se situent à 0,22 sur l'échelle de l'autonomie quand les plus jeunes se situent près de $0{ }^{25}$.

\section{La formation, source de revenus}

La formation fournit, dans l'immédiat, un complément aux revenus familiaux ou de l'argent de poche ; elle est parfois l'unique source de revenus. L'argent n'est pas affiché comme étant moteur dans l'engagement en formation. Si l'on cherche à distinguer le rapport à l'argent selon les différentes trajectoires, on relève cependant que c'est parmi les jeunes qui n'ont rien déclaré comme activité ou qui n'ont pas connu d'autres expériences que celle de la formation que la question d'argent pourrait bien être avancée, si elle était moins taboue ${ }^{26}$. Quoi d'autre pour gagner un peu d'argent ?...

\footnotetext{
${ }^{24}$ On compare les moyennes de la dimension Espoir stage du temps 1 de l'enquête à celles du temps 2 : elles apparaissent significativement différentes selon le $|\mathrm{t}|$ de Student au seuil $\mathrm{P}<0,001$.

25 Ecart significatif au seuil $\mathrm{P}<0,001$.

${ }^{26}$ Leur position sur l'échelle de valeur Argent, bien que négative, est supérieure à celle affichée par ceux qui ont déjà travaillé ou perçoivent des indemnités de chômage notamment.
}

Notons que la question d'argent prend significativement de l'importance au temps 2 de l'enquête. La perspective de la fin de la formation ferait-elle craindre une perte ou une baisse des revenus ? L'espoir d'embauche s'amenuisant, cette question prend-elle de l'importance ? Vient-elle signifier un désinvestissement de la formation? Ne resterait-il, pour certains, qu'une motivation financière en fin de formation?

\section{La formation, soutien dans le changement}

Non seulement la formation n'est pas vécue sur le mode de la contrainte - même si certains reconnaissent par ailleurs être dans l'obligation de suivre une formation - mais de manière générale, l'attente est forte à l'égard des organismes de formation dans le soutien à la réalisation de nouveaux projets. Les expériences accumulées, y compris les expériences de chômage long, n'entament pas l'espoir d'un changement. Il est attendu de la formation qu'elle change quelque chose au sort de ses bénéficiaires volontaires ou non ; et ce, quels que soient les parcours biographiques ${ }^{27}$. Un sous-groupe se démarque dans cette attente forte et homogène, en affichant encore plus de détermination que les autres : ce sont les femmes en milieu rural $^{28}$. La campagne aurait un effet amplificateur sur les difficultés de retour ou d'accès à l'emploi et à la formation pour les femmes (Brunhes-Glamcevski, 2005). L'analyse des expériences et des rapports à la formation doit prendre en compte non seulement les contextes géographiques (Levené \& Dubus, 2010) mais aussi la manière dont sont distribués les rôles sociaux dans notre société.

\section{La formation pour développer le lien social}

La formation volontaire, encouragée ou contrainte, qu'elle soit destinée à une meilleure maitrise des savoirs de base ou à une (re)mobilisation ou encore à l'aide à la définition d'un projet professionnel, semble

\footnotetext{
${ }^{27} \mathrm{La}$ moyenne des positions sur l'échelle de la dimension Changement est de 0,36, avec un écart type de 0,22.

${ }^{28}$ La recherche a pris en compte les contextes géographiques des enquêtés pour l'analyse des représentations et attentes vis-à-vis de la formation. Les femmes rurales se situent à 0,49 sur l'échelle du Changement.
} 
avoir une fonction de lien social ; cela s'explique sans doute par ses contenus davantage basés sur le développement de compétences ou de savoirs êtres que de savoir-faire ou de connaissances techniques ou générales. Les attentes en termes de sociabilité se distinguent quelque peu selon les expériences. Les femmes ayant consacré jusqu'alors leur temps à l'éducation de leur(s) enfant(s) ressentent un besoin de contacts que l'inscription en MSB doit ou semble venir satisfaire. Elles rejoignent en cela les plus âgés de la population que la perte d'emploi expose à une disqualification sociale (Paugam, 2000); ils attendent généralement plus de la formation, en termes de développement de lien social, que les moins de vingt ans qui vivent généralement chez leurs parents et qui sont relativement socialisés dans leurs quartiers ${ }^{29}$.

\section{La formation comme réponse au besoin d'être utile}

La formation contribue au développement d'un sentiment d'utilité : la moyenne des positions sur l'échelle de cette dimension est de 0,26 . La formation satisfait un besoin d'utilité qui va de pair avec un besoin d'apprendre et d'évoluer ${ }^{30}$. L'idée d'être inutile ou de "ne plus être dans la course $»^{31}$ est d'autant plus insupportable que les enquêtés possèdent des ressources qui, «normalement », prédisent une bonne insertion : le niveau de qualification est déterminant. Le Sentiment d'utilité obtient un score moyen de 0,41 dans le groupe des plus qualifiés (niveau 4) de la population interrogée; un score de 0,25 dans le groupe des moins qualifiés (niveau 5 et infra 5 ).

De manière générale, ce sentiment est davantage exprimé parmi les plus éloignés de l'emploi : ceux qui n'ont connu jusqu'alors que des situations professionnelles précaires, les chômeurs de longue durée ou encore les plus vulnérables sans qualification et sans expérience professionnelle $(0,29$ sur l'échelle du Sentiment d'utilité). Ils se distinguent à cet égard, d'une part, de ceux qui font état d'une « carrière » professionnelle longue parmi les plus âgés, et d'autre

\footnotetext{
${ }^{29}$ Ecart significatif de 0,09 sur l'échelle de la Sociabilité.

30 " un emploi pour se sentir utile», « un emploi pour continuer à apprendre et évoluer » sont des items proposés à l'appréciation des enquêtés et regroupés sous la dimension Sentiment d'utilité.

${ }^{31}$ Propos recueillis auprès d'un stagiaire au cours de l'enquête.
}

part de mères de famille $(0,19)$. Ces derniers ont en commun une intégration professionnelle, y compris indirecte pour les femmes dont l'intégration familiale est souvent renforcée par une intégration professionnelle liée à leurs conjoints en emploi (6 femmes vivant en couple sur 10 déclarent un conjoint en emploi $)^{32}$.

Cette nécessité de satisfaire un besoin d'utilité est non négligeable et révèle une profonde détresse. Elle ne doit cependant pas justifier la transformation des dispositifs de formation en «dispositifs de bienveillance », pour reprendre l'expression de Foucart (2009), en faisant des activités occupationnelles et socialisatrices leur unique objectif.

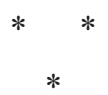

En échec scolaire, orientés dans des filières sans débouchés, assignés à des postes de travail non qualifiés, sans perspective d'évolution ou de formation interne, aux horaires flexibles et aux conditions de travail souvent pénibles, exposés aux risques du chômage et inscrits dans des formations non qualifiantes, les adultes rencontrés en parcours d'insertion pâtissent d'une organisation sociale qui rend improbable le droit, pourtant fondamental, de chacun à un travail. Pour autant, la situation de formation est investie plutôt positivement par ses bénéficiaires : la disponibilité des formateurs, les relations qui peuvent s'y instaurer, les activités proposées ${ }^{33}$, les stages en entreprise détournent d'un quotidien morose. Ils font renaître (un temps) l'espoir d'une vie meilleure, pas nécessairement liée à l'emploi, qui n'apparaît pas toujours comme l'objectif premier.

Au-delà, les dispositifs mis en place répondent-ils aux intérêts des adultes peu qualifiés ? Concourent-ils à

\footnotetext{
${ }^{32}$ Il est fait ici référence à Barel (1990) cité par Castel (1995, p. 413) pour qui le travail fait fonction de " grand intégrateur»: le travail étant "un inducteur » qui traverse les autres formes d'intégration : scolaire, familiale, sociale, culturelle, etc. Les femmes remplissant un rôle familial et social par l'éducation de leurs enfants affichent une identité de mères de famille. Leurs conjoints en emploi enrichissent ces identités familiale et sociale de valeurs et reconnaissances liées au travail. Elles sont mères et épouses de... La reconstitution des parcours de femmes dans la troisième partie du questionnaire s'organise très souvent, en l'absence d'expériences professionnelles propres, autour d'événements familiaux et liés à la carrière professionnelle du conjoint.

${ }^{33}$ Témoignages des chercheurs-enquêteurs.
} 
restaurer leur employabilité ? On peut s'interroger sur la pertinence des programmes proposés, et sur les temps et les moyens alloués à la formation; d'autant plus que la circulation observée de l'un à l'autre des dispositifs, les attentes exprimées et les espoirs déçus révèlent un réel décalage entre les intentions politiques et les moyens mis en œuvre.

Certes, le terme employabilité n'a pas été prononcé par les formateurs dans les centres de formation visités ; cependant, la seule référence qui vaille est l'entreprise et on observe une nette tendance à expliquer le chômage par l'inadaptation sociale des individus : «Il faut d'abord qu'il apprenne à se lever à l'heure, à prendre les transports en commun seul ou encore à se laver régulièrement avant de prétendre à l'emploi [ou à la simulation d'emploi par le stage]», a-t-on entendu dire à propos d'un stagiaire. Le stage doit dans certains cas se mériter, ce qui sans doute contribue à le rendre si attractif et... si décevant.

La remise à niveau doit également, dans l'esprit de certains formateurs, précéder la recherche d'emploi. Or l'enquête "Information Vie quotidienne » de l'INSEE ${ }^{34}$ a montré que, parmi les adultes qui travaillent, la part de ceux qui connaissent de graves difficultés à l'écrit est de $7 \%$. L'enquête menée en Nord Pas de Calais et Champagne-Ardenne révèle la forte présence de salariés parmi les stagiaires inscrits en réapprentissage des savoirs de base. Pourtant, on préférera souvent faire de l'illettrisme une problématique sociale plutôt qu'une problématique d' " échec scolaire » au sens d'échec de l'École. Envisager l'illettrisme sous l'angle social, c'est le traiter par le biais des dispositifs de lutte contre l'exclusion, au risque de ne pas proposer de solutions adaptées (Benyayer \& Boudol, 2003).

L'exigence d'une définition de projet professionnel, en programme d'orientation (qui porte le nom de « définition de projet professionnel »), ou personnel, en programme de mobilisation, apparaît presque indécente quand on mesure la marge de liberté très réduite dont disposent objectivement ces jeunes ou moins jeunes, davantage préoccupés par leur situation

\footnotetext{
${ }^{34}$ L'enquête « Information Vie quotidienne » de l'INSEE a mesuré les compétences en lecture, écriture, calcul d'un échantillon représentatif (10 000 personnes) de la population âgée de 18 à 65 ans, vivant en France métropolitaine.
}

présente insécurisante (Castra, 2003). La formation les protège temporairement d'un avenir menaçant, autant par l'occupation qu'elle procure que par les subsides qu'elle apporte.

Enfin, la circulation des stagiaires observée d'un dispositif à l'autre illustre une certaine forme de brouillage des objectifs, inhérent au "marché du conseil aux chômeurs » (Castra, 2003) enfermant ces derniers dans «le statut d'inséré permanent» : la pré-qualification prépare difficilement à la qualification, le réapprentissage des savoirs de base accueille des étrangers qualifiés, la mobilisation et l'orientation n'offrent pas les débouchés annoncés... Assisterait-on à une forme de dérive de la perspective économiste du deuxième âge de l'insertion ? Les besoins de qualification des « inemployables » semblent, dans ce contexte, loin d'être satisfaits. À leur manière, ils l'expriment.

Les « stagiaires permanents » soufrent depuis longtemps d'un « déficit d'intégration », pour reprendre l'expression de Castel (1995), en raison des quartiers qu'ils habitent, de leurs difficultés scolaires, des écoles qu'ils fréquentent, de parents peu qualifiés ou qui n'ont pu céder leur place à leurs fils ou filles, leurs usines ayant fermé : $45 \%$ des enquêtés en effet déclarent un père ouvrier qualifié...

Les politiques d'insertion, en ciblant des populations particulières, signent en quelque sorte la faillite de l'intégration de certains groupes sociaux « en se situant en deçà des ambitions des politiques intégratives universalistes $»^{35}$ (Castel, 1995, p. 418). Dans ce contexte, «les discours et les efforts portant sur l'employabilité [et développés dans les centres de formation par les formateurs et les bénéficiaires] masquent [difficilement] une exclusion de fait des inemployables »(Boltanski \& Chiapello, 1999, p. 312). Il y aurait lieu de se demander si le but n'est pas de les convaincre que « la société est inégale par devoir économique $»^{36}$ (Ebersold, 2001, p. 187)?

\footnotetext{
${ }^{35}$ Comme celles qui ont conduit à la mise en place de la Sécurité sociale.

${ }^{36}$ Si l'on considère que la « loi du marché » s'impose, les inégalités sociales, scolaires, économiques sont inévitables. La théorie de la dualisation de la société d'A. Touraine s'inscrit dans cette perspective.
} 
Alaluf M. (2000), Dictionnaire du prêt à penser, Bruxelles, Evo.

Arrighi J.-J., Gasquet C. (2010), « Orientation et affectation : la sélection dans l'enseignement professionnel du second degré », Formation Emploi, 109, pp. 99-112.

Arrighi J.-J, Gasquet C., Joseph O. (2009), "L'insertion des sortants de l'enseignement secondaire. Des résultats issus de l'enquête génération 2004 », NEF Céreq, 42.

Barel Y. (1990), «Le grand intégrateur », Connexions, 56.

Bartholome C., Vrancken D. (2005), « L'accompagnement : un concept au cœur de l'état social actif. Le cas des pratiques d'accompagnement des personnes handicapées ", Pensée plurielle, 10, pp. 85-95.

Baud P., Bourgeat S., Bras C. (2008), Dictionnaire de géographie, Paris, Hatier.

Bautier E., Terrail J.-P., Branca-Rosoff S.et al. (2002), Décrochage scolaire : genèse et logique des parcours, Rapport de recherche pour la DPD/MEN, $327 \mathrm{p}$.

Bec C. (1999), « Quand l'assistance se substitue au salaire », Alternatives économiques, 175.

Benyayer C, Boudol N. (2003), « L'illettrisme : beaucoup de mots pour qui ? ", Education permanente, 156, pp. 163-172.

Boltanski L., Chiapello E. (1999), Le nouvel esprit du capitalisme, Paris, Gallimard.

Brunhes-Glamcevski B. (2005), " La femme en milieu rural et l'influence de son milieu de vie ». Colloque FAIRE CAMPAGNE, Rennes, 17-18 mars.
Castel R. (1995), Les métamorphoses de la question sociale, une chronique du salariat, Paris, Fayard.

Castra D. (2003), L'insertion professionnelle des publics précaires, Paris, PUF.

Delort A. (2010), « La dépense nationale pour la formation professionnelle continue et l'apprentissage, en $2008 »$, Dares Analyses, 073.

Demunter C. (1999), « Intérêts de classe et qualification sociale », Contradictions, Paris, L'Harmattan.

Dietrich A., Jouvenot C., Lenain M.-C. (2007), « L'employabilité sous conditions : le cas Metaleurop ", Education permanente, 170, pp. 130-142.

Dubar C. (2004), La formation professionnelle continue, Paris, La Découverte.

Ebersold S. (2001), La naissance de l'inemployable, Presses universitaires de Rennes.

Foucart J. (2009), « L'accompagnement : dispositif de bienveillance et construction transactionnelle », Pensée plurielle, 22, pp. 13-27.

Franssen A. (2008), « De la critique de l'activation à la démocratisation de l'action sociale ", Politiques, hors série ${ }^{\circ} 9$, pp. $70-83$.

Gasquet C. (2003), « Les jeunes "sans qualification", un groupe hétérogène, des parcours d'insertion divers », Bref Céreq, 202.

Hagnere C. (2004), « Le contrat d'insertion revenu minimum d'activité. Contexte et perspectives ", Revue de l'OFCE, 88, pp. 103-118.

Janosz M. (2000), « L'abandon scolaire chez les adolescents : perspective nord-américaine », VEI Enjeux, 122, pp. 105-127. 
Janosz M., Leblanc M., Boulerice B. (1998), « Consommation de psychotropes et délinquance : de bons prédicteurs pour l'abandon scolaire ?", Criminologie, XXXI, 1, pp. 87-107

Jauneau Y. (2009), " Les employés et ouvriers non qualifiés », INSEE Première, 1250.

Levene T., Dubus A. (2010), Expériences, parcours, contextes résidentiels et rapports à la formation des adultes peu qualifiés : http://halshs.archives-ouvertes. fr/halshs-00529887/fr/

Mauger G. (2001), « Les politiques d'insertion. Une contribution paradoxale à la déstabilisation du marché du travail », Actes de la recherche en sciences sociales, 136-137, pp. 5-14
Paugam S. (2000), La disqualification sociale : essai sur la nouvelle pauvreté, Paris, PUF

Renahy N. (2006), Les Gars du coin. Enquête sur une jeunesse rurale, Paris, La Découverte.

Rose J. (2009) « La "non qualification" Question de formation, d'emploi ou de travail ? », Net.doc Céreq, 53.

Roche S. (2001), La délinquance des jeunes, les 13-19 ans racontent leurs délits, Paris, Seuil.

Vergne F. (2006), « Un autre regard sur l'orientation et la relation formation-emploi », Actualité de la formation permanente, 202, pp. 59-64.

\section{Résumé}

\section{Les politiques d'insertion : quelle pertinence pour les « inemployables »? \\ Thérèse Levené}

S'inscrivant d'abord dans une perspective éducative, les politiques publiques soutiennent aujourd'hui essentiellement les initiatives d'insertion par l'économique. Le chômage serait dorénavant imputé à "l'inemployabilité » des personnes et les dispositifs d'insertion ont pour mission d'améliorer l'attractivité professionnelle des sans-emploi. Quels traitements proposent-ils aux « inemployables »? Nous nous appuyons sur une enquête menée dans le quart nord-est de la France auprès d'adultes en difficulté avec l'écrit, inscrits dans des dispositifs de (ré)insertion professionnelle. Elle met à jour leurs caractéristiques et leurs attentes et interroge la pertinence de l'offre d'insertion en termes de développement de leur employabilité.

\section{Mots clés}

Politique publique, insertion par l'économique, chômeur, employabilité, réinsertion professionnelle Journal of Economic Literature: J 24, J 68 